\title{
Case report: a 37-year-old male with telangiectasias, polycythemia vera, perinephric fluid collections, and intrapulmonary shunting
}

\author{
Javed Khan ${ }^{1}$ and David B Sykes ${ }^{2^{*}}$
}

\begin{abstract}
Background: The TEMPI syndrome was recently described in 2011, and is characterized by the constellation of five hallmarks: Telangiectasias, Erythrocytosis and elevated Erythropoietin, Monoclonal gammopathy, Perinephric fluids collections, and Intrapulmonary shunting. The underlying pathophysiology is unknown, though it has been postulated that the monoclonal gammopathy may play a causal role.
\end{abstract}

Case presentation: A 37-year-old non-smoking male presented to our institution with a fever and the sensation of fullness in the right flank. His exam was notable for telangiectasias, clubbing of the fingernails, plethora, and a palpable bulge in the right flank. Renal ultrasound demonstrated bilateral perinephric fluid collections. Laboratory evaluation revealed erythrocytosis with low serum erythropoietin, and testing for the JAK2V617F mutation was positive, confirming a diagnosis of polycythemia vera. Though his room air saturation was normal at rest, it decreased dramatically with exercise, felt to be secondary to microscopic intrapulmonary shunting.

The patient's presentation is very similar to that of the TEMPI syndrome, a very rare syndrome of which there have been six published cases. In contrast to the TEMPI syndrome where the erythrocytosis is driven by highly elevated serum erythropoietin, our patient was found to have polycythemia vera. Also in contrast to the other patients with TEMPI syndrome, our patient did not have an identifiable monoclonal gammopathy.

Our patient responded to treatment with hydroxyurea. His erythrocytosis, perinephric fluid collections, and telangiectasias resolved over the course of six months. The intrapulmonary shunting has continued to gradually improve with treatment, suggesting that this is an entirely reversible process.

Conclusion: Our case is the first to describe the combination of polycythemia vera, telangiectasias, perinephric fluid collections, and intrapulmonary shunting. The presentation is highly similar to the previously described TEMPI syndrome, though calls into question the potential importance of the monoclonal gammopathy. Our patient demonstrated a response to treatment with hydroxyurea, while patients with the TEMPI syndrome have shown responses to plasma-cell directed therapies such as bortezomib.

Keywords: Erythrocytosis, Erythropoietin, Monoclonal gammopathy, Perinephric fluid, Polycythemia, TEMPI

\section{Background}

The description of the TEMPI syndrome, and a small case series of three patients, was recently published by Sykes et al. in the New England Journal of Medicine [1]. The syndrome was designated TEMPI by the authors based on five key characteristics:

\footnotetext{
* Correspondence: dbsykes@partners.org

${ }^{2}$ Division of Hematology, Massachusetts General Hospital Cancer Center, 55 Fruit Street, Boston, MA 02114, USA

Full list of author information is available at the end of the article
}

- Telangiectasias

- Erythrocytosis \& elevated serum Erythropoietin

- Monoclonal gammopathy

- Perinephric fluid collections

- Intrapulmonary shunting

Further review of the literature identified three additional patients [2-4], but failed to reveal a pathophysiological understanding of this syndrome. Two additional patients have also been identified, but not published, bringing the 
total to eight known living patients with the TEMPI syndrome (Sykes DB, personal communication).

\section{Case presentation}

A 37 year-old male non-smoking farmer with no prior medical history initially presented to his physician with concerns of flank pain and fever. On interview, he had noted telangiectasias (he described them as 'small boils') for approximately one year prior to presentation. He was febrile to a temperature of $102^{\circ} \mathrm{F}$. He had normal room air oxygen saturation at rest (98\%). His physical exam was notable for plethora, clubbing in the nails of the hands, telangiectasias on the back (Figure 1) and a palpable mass in the right abdomen. Testing of the stool for occult blood was negative.

An abdominal ultrasound revealed perinephric fluid collections, larger on the right side than on the left side. A computed tomography scan of the abdomen confirmed the ultrasound findings and demonstrated a right-sided perinephric fluid collection measuring $10 \mathrm{~cm} \times 12 \mathrm{~cm} \times$ $17 \mathrm{~cm}$ as well as a very small collection on the left side (Figure 2) and mild splenomegaly. Of particular note
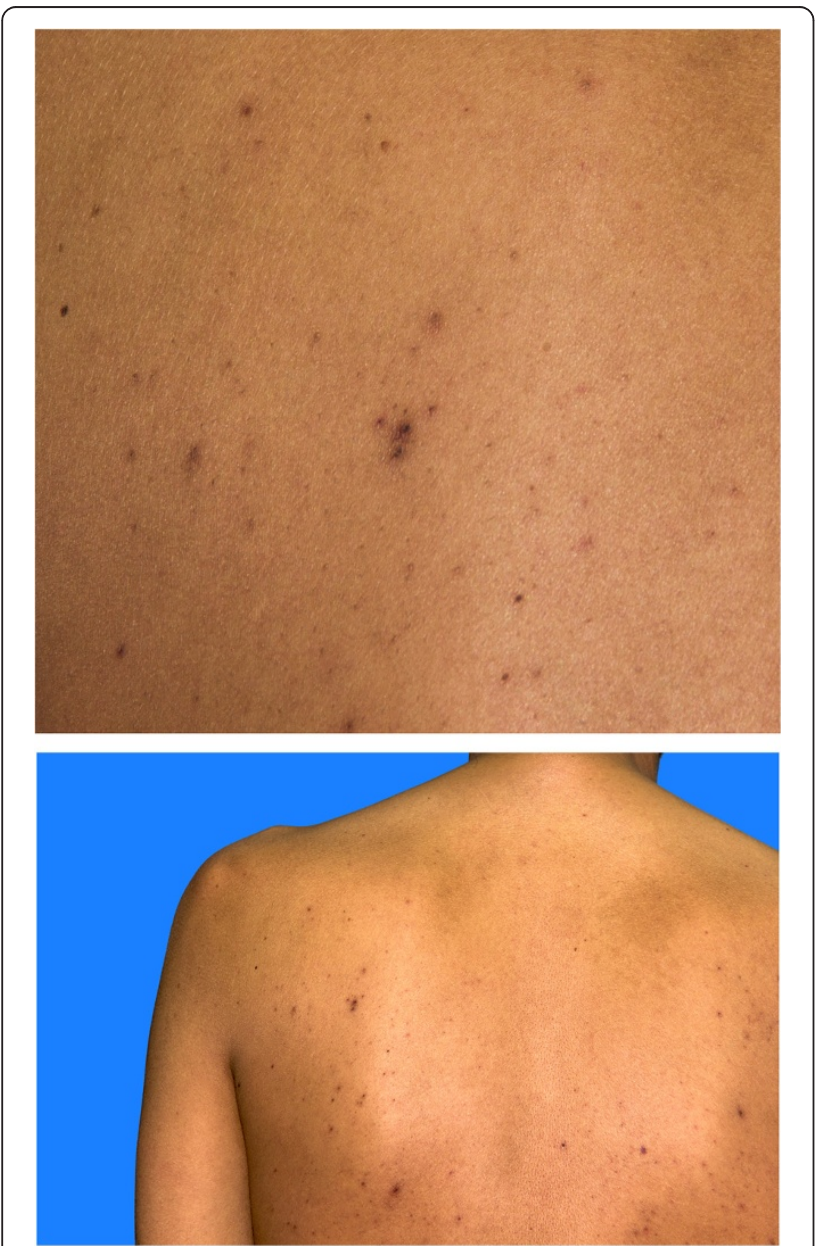

Figure 1 Telangiectasias were noted on the back and trunk.

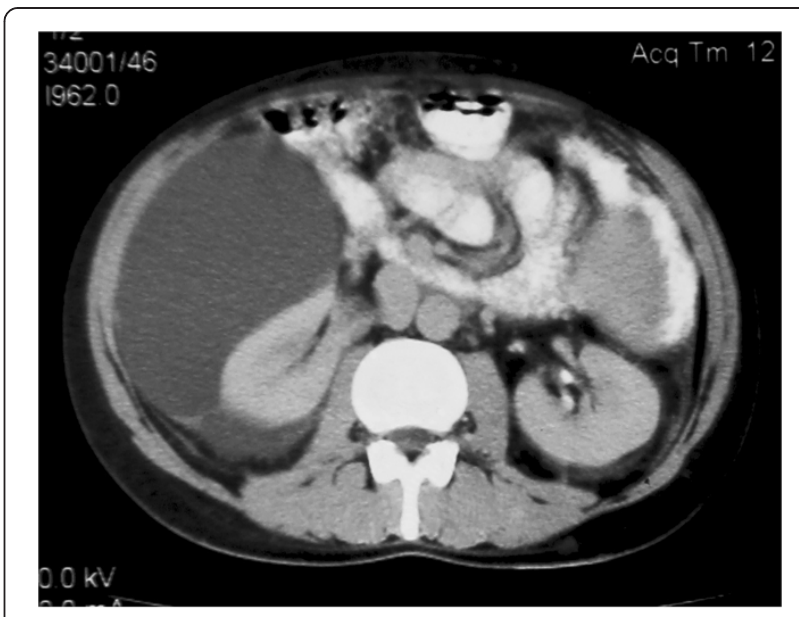

Figure $2 \mathrm{~A}$ contrast enhanced CT scan of the abdomen demonstrates showing a large right-sided perinephric fluid collection.

was the lack of renal cysts and the lack of any intraparenchymal fluid collections.

The patient underwent an extensive laboratory evaluation (Table 1). His complete blood count demonstrated polycythemia and a neutrophil-predominant leukocytosis. The serum electrolytes were normal; BUN and creatinine were slightly elevated. The evaluation of his erythrocytosis revealed a suppressed serum erythropoietin of 2 $\mathrm{mIU} / \mathrm{ml}$ (normal range $4-17 \mathrm{mIU} / \mathrm{ml}$ ) and a heterozygous JAK2V617F mutation consistent with a diagnosis of polycythemia vera. Our patient did not have a history of thrombosis and did not describe any symptoms consistent with erythromelalgia.

His red blood cells were initially microcytic (MCV $67 \mathrm{fl}$ ), though this corrected into the normal range with oral iron supplementation. A hemoglobin electrophoresis was not performed.

The serum protein electrophoresis was normal, and immunofixation did not reveal any monoclonal bands. Evaluation of the urine did not reveal any Bence-Jones protein. The serum uric acid was slightly elevated, this was felt to be due to increased RBC turnover in the setting of polycythemia.

The patient's urinalysis was normal. He underwent aspiration of the right-sided perinephric fluid collection and a drainage catheter was left in place. Examination of the fluid revealed a clear fluid with few RBC and few inflammatory cells. Cytology revealed no malignant cells. The fluid had very low levels of protein, glucose, and triglycerides. The fluid amylase was $15 \mathrm{U} / \mathrm{L}$. The fluid creatinine was $0.74 \mathrm{mg} / \mathrm{dL}$, arguing against the possibility of a urinoma.

He next underwent evaluation of his exercise tolerance and oxygen saturation. His room air oxygen saturation was $95 \%$ at baseline but decreased to $75 \%$ after 2 minutes of 
Table 1 Laboratory evaluation

\begin{tabular}{|c|c|c|}
\hline Parameter & Result & Normal range \\
\hline WBC (TLC) & 12,000 & $4000-11000 / \mathrm{cmm}$ \\
\hline $\mathrm{RBC}$ & 9.94 & 4.4-5.5 million/cmm \\
\hline $\mathrm{Hb}$ & 19.6 & $14-18 \mathrm{gm} / \mathrm{dl}$ \\
\hline $\mathrm{HCT}(\mathrm{PCV})$ & 66.7 & $40-45 \%$ \\
\hline MCV & 67 & $80-96 \mathrm{fL}$ \\
\hline $\mathrm{MCH}$ & 19.7 & 27-32 pgm \\
\hline $\mathrm{MCHC}$ & 29.4 & $30-35 / \mathrm{dl}$ \\
\hline Platelets & 572,000 & $150,000-400,000 / \mathrm{cmm}$ \\
\hline \multicolumn{3}{|l|}{ Differential (DLC) } \\
\hline Neutrophils & $90 \%$ & \\
\hline Lymphocytes & $08 \%$ & \\
\hline Monocytes & $02 \%$ & \\
\hline Eosinophils & $00 \%$ & \\
\hline \multicolumn{3}{|l|}{ RBC MORPHOLOGY } \\
\hline Anisocytosis & +ive & \\
\hline Hyphochromia & +ive & \\
\hline Microcytosis & +ive & \\
\hline Sodium & 133 & $135-145 \mathrm{mmol} / \mathrm{L}$ \\
\hline Potassium & 4.7 & $3.4-4.8 \mathrm{mmol} / \mathrm{L}$ \\
\hline Chloride & 105 & $100-108 \mathrm{mmol} / \mathrm{L}$ \\
\hline Blood Urea Nitrogen & 37 & $15-40 \mathrm{mg} / \mathrm{dl}$ \\
\hline Creatinine & 1.9 & $0.9-1.3 \mathrm{mg} / \mathrm{dl}$ \\
\hline Glucose & 100 & $70-110 \mathrm{mg} / \mathrm{dl}$ \\
\hline Total Proteins & 5.7 & $6-7 \mathrm{~g} / \mathrm{dl}$ \\
\hline Albumin & 3.5 & $3.2-5 \mathrm{~g} / \mathrm{dl}$ \\
\hline Globulin & 2.2 & $1.8-3.6 \mathrm{~g} / \mathrm{dl}$ \\
\hline Bilirubin (total) & 1.2 & $0.0-1.0 \mathrm{mg} / \mathrm{dl}$ \\
\hline $\mathrm{ALT}(\mathrm{SGPT})$ & 17 & $10-55 \mathrm{U} / \mathrm{L}$ \\
\hline AST (SGOT) & 14 & $10-40 \mathrm{U} / \mathrm{L}$ \\
\hline Uric acid & 9 & $3.4-7.2 \mathrm{mg} / \mathrm{dl}$ \\
\hline PT & 14 & (Control = $12 \mathrm{sec})$ \\
\hline aPTT & 34 & (Control = $32 \mathrm{sec}$ ) \\
\hline $\mathrm{HBsAg}$ & Non-Reactive & Non-Reactive \\
\hline Anti HCV Abs & Non-Reactive & Non-Reactive \\
\hline HIV & Non-Reactive & Non-Reactive \\
\hline
\end{tabular}

exercise on the treadmill, at which point the test was stopped. The patient's ECG and echocardiogram were normal. Microscopic intrapulmonary shunting was suspected.

The abdominal CT scan and voiding urogram confirmed the large right-sided perinephric fluid collection. The collection compressed the right kidney though the excretion of dye was normal. The left kidney showed only a small amount of perinephric fluid. The CT urography showed no connection of the fluid collection with renal collecting system.
Cultures of urine, blood, and perinephric fluid showed no aerobic or anaerobic growth. Examination of thick and thin blood smears showed no malarial parasites.

Testing for Anti-nuclear antibodies (ANA), Anti-Smooth Cell Antibodies (ASMA), and Anti-mitochondrial Antibodies (AMA) was negative.

The patient was seen in consultation by radiology, nephrology, surgery, and medical teams. He underwent therapeutic phlebotomy to remove one unit of blood. He then initiated therapy with hydroxyurea (500 mg given twice daily by mouth). Over the first two weeks of treatment, daily drainage output decreased from $1500 \mathrm{ml}$ daily to $50 \mathrm{ml}$ daily, and the drain was removed.

The patient was discharged. On his first follow-up visit (approximately three months) his hemoglobin was $17.5 \mathrm{mg} / \mathrm{dl}$ and an ultrasound confirmed that his perinephric fluid collections had resolved. On his second follow-up visit (approximately six months) his hemoglobin was $16.4 \mathrm{mg} / \mathrm{dl}$. His telangiectasias had also almost completely resolved and he felt that his exercise tolerance was improved though formal exercise pulse oximetry was not performed.

\section{Conclusions}

Our patient presented with four of the five hallmark characteristics of the TEMPI syndrome, including telangiectasias, erythrocytosis, perinephric fluid collections, and intrapulmonary shunting. Of particular note, our patient lacked the monoclonal gammopathy common to patients with TEMPI syndrome. To date, 8 living patients have been identified with the TEMPI syndrome, all possess a monoclonal gammopathy (Sykes DB, personal communication). Furthermore, in contrast to these other eight patients, our patient's erythrocytosis was due to polycythemia vera rather than to an elevated serum erythropoietin level.

Our patient appears to have a unique constellation of symptoms, not previously described in the literature. The pathophysiology underlying the telangiectasia formation and intrapulmonary shunting is not clear. In patients with the TEMPI syndrome, the authors postulated that the monoclonal gammopathy might be playing a causal role [5]. However, our patient presented with both telangiectasias and intrapulmonary shunting in the absence of a monoclonal gammopathy, arguing against this hypothesis.

The patients with TEMPI syndrome also have a characteristically elevated serum erythropoietin level, elevated to 10-500 times the upper limit of normal. However, our patient had a low serum erythropoietin. This also argues against the possibility that EPO is acting as a proliferative signal in the abnormal blood vessel formation (telangiectasia and microscopic intrapulmonary shunting) that is characteristic of patients with the TEMPI syndrome. 
In patients with the TEMPI syndrome, partial or complete responses have been seen upon treatment with the proteasome inhibitor bortezomib (Kwok 2012 [3] and Schroyens 2012 [5]). Our patient has had a response, in terms of his erythrocytosis, telangiectasias, and perinephric fluid collections, to simple treatment with hydroxyurea. We have not yet seen a complete response in terms of his intrapulmonary shunting as manifested by his decreased exercise oxygen saturation, though this may simply take longer to reverse.

International collaboration and modern medical search engines have made it possible to identify collections of ultra-rare patients such as ours. We hope that the continued description of these patients will eventually lead to an understanding of their disease pathophysiology and provide a rational means of disease treatment.

\section{Consent}

Written informed consent was obtained from the patient for publication of this case report and for the accompanying images. A copy of the written consent is available for review.

\section{Abbreviation}

TEMPI: Telangiectasias, Erythrocytosis and elevated Erythropoietin, Monoclonal gammopathy, Perinephric fluids collections, and Intrapulmonary shunting.

\section{Competing interests}

The authors declare that they have no competing interests.

\section{Authors' contributions}

JK identified the case, and led the workup of the patient, including making the diagnosis and initiating the treatment of the patient. JK also helped to draft the manuscript. DBS consulted on the diagnostic workup and care of the patient. DBS also helped to draft the manuscript. Both authors read and approved the final manuscript

\section{Acknowledgments}

The authors would like to acknowledge Dr. Wilfried Schroyens for his critical appraisal of the manuscript, Dr. Nisar Ahmad (Registrar Surgical "A" unit) for his help in identifying the case, and Dr. Mushtaq Ahmad (Resident Surgical "E" unit) for his financial help to the patient.

\section{Author details}

'Room 91 Old Doctors Hostel, Khyber Teaching Hospital Peshawar, Peshawar 25000, Pakistan. ${ }^{2}$ Division of Hematology, Massachusetts General Hospital Cancer Center, 55 Fruit Street, Boston, MA 02114, USA.
Received: 10 January 2014 Accepted: 20 June 2014

Published: 22 July 2014

\section{References}

1. Sykes DB, Schroyens W, O'Connell C: The TEMPI syndrome-a novel multisystem disease. N Engl J Med 2011, 365(5):475-477.

2. Mohammadi F, Wolverson MK, Bastani B: A new case of TEMPI syndrome. Clin Kidney J 2012, 5(6):556-558. first published online November 1, 2012

3. Kwok M, Korde N, Landgren O: Bortezomib to treat the TEMPI syndrome. N Engl J Med 2012, 366(19):1843-1845.

4. Ryden A, Wei K, Rodriguez R, Mahrer T: Too much blood: a case of the newly described TEMPI syndrome. Chest 2013, 1:144. (4_MeetingAbstracts): 927A.

5. Schroyens W, O'Connell C, Sykes DB: Complete and partial responses of the TEMPI syndrome to bortezomib. N Engl J Med 2012, 367(8):778-780.

doi:10.1186/2052-1839-14-11

Cite this article as: Khan and Sykes: Case report: a 37-year-old male with telangiectasias, polycythemia vera, perinephric fluid collections, and intrapulmonary shunting. BMC Hematology 2014 14:11.

\section{Submit your next manuscript to BioMed Central and take full advantage of:}

- Convenient online submission

- Thorough peer review

- No space constraints or color figure charges

- Immediate publication on acceptance

- Inclusion in PubMed, CAS, Scopus and Google Scholar

- Research which is freely available for redistribution 